\title{
Erratum: Screening and nonlocal correlations in the extended Hubbard model from self-consistent combined $G W$ and dynamical mean field theory [Phys. Rev. B 87, 125149 (2013)]
}

\author{
Thomas Ayral, Silke Biermann, and Philipp Werner \\ (Received 18 November 2016; published 22 December 2016)
}

DOI: 10.1103/PhysRevB.94.239906

Section V, step 5(a) of this paper contains two errors in the description of the many-body self-energy that was used in the combined $G W+$ extended dynamical mean field theory (EDMFT) calculations to obtain the results plotted in Figs. 12, 13, 14, and 15. The first one is a typo, while the second one is more fundamental and also affects the $G W+$ EDMFT results in Refs. [1,2]. While the results in these papers are valid, the $G W+$ EDMFT data correspond to a slightly different model than indicated, due to the omission of the nonlocal Fock term.

First, the sign in the second term of the equation for the $G W$ self-energy (the second line in the second expression of point $5(\mathrm{a})$ in Sec. V), is wrong. The correct expression is

$$
\Sigma^{G W}(\mathbf{k}, \tau)=-\sum_{\mathbf{q}} G(\mathbf{q}, \tau) W^{c}(\mathbf{k}-\mathbf{q}, \tau)-\sum_{\mathbf{q}} G_{\mathbf{q}+\mathbf{k}}\left(\tau=0^{+}\right) v_{\mathbf{q}}
$$

The second issue concerns the nonlocal Fock term, which we erroneously assumed to vanish in this work and in Refs. [1,2]. While this term should be, and is, included in the equations describing the $G W+$ EDMFT formalism, it was set to zero in the actual calculations. Hence the results in Figs. 2, 3(a), 3(b), and 4 of Ref. [1], Figs. 12, 13, 14, and 15 of this paper, and Figs. 11, 12,13 , and 14 of Ref. [2] were obtained with the $G W$ self-energy

$$
\Sigma^{G W}(\mathbf{k}, \tau)=-\sum_{\mathbf{q}} G(\mathbf{q}, \tau) W^{c}(\mathbf{k}-\mathbf{q}, \tau),
$$

and should thus be considered as results for a model in which the effect of the nonlocal Fock term is already included at the level of the one-particle dispersion.

While the nonlocal Fock term vanishes trivially in the limit $V=0$, it is nonzero and static for $V>0$. Its effect is a band widening that can substantially increase the one-particle bandwidth, and does so in an explicitly $V$-dependent and implicitly $U$-dependent manner. Its quantitative effect on the dispersion is illustrated in Fig. 1, which shows results for $U=2, V=0.4$ and
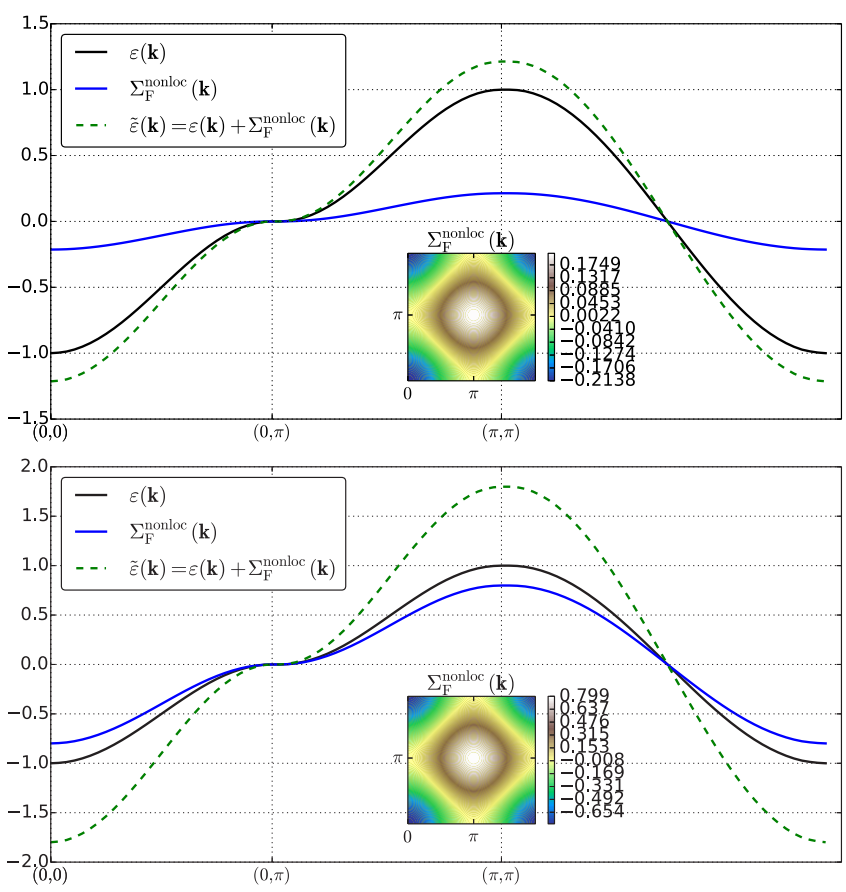

FIG. 1. Bare dispersion $\varepsilon(\mathbf{k})$ (solid black lines), nonlocal Fock self-energy $\Sigma_{\mathrm{F}}^{\text {nonloc }}(\mathbf{k})$ (solid blue line), and renormalized dispersion $\tilde{\varepsilon}(\mathbf{k})$ (dashed green line) along a high-symmetry path in the Brillouin zone in the $G W+$ EDMFT method. Inset: Color plot of $\Sigma_{\mathrm{F}}^{\text {nonloc }}(\mathbf{k})$ in the first Brillouin zone. The top panel shows data for $U=2.0$ and $V=0.4$, the bottom panel for $U=3.0$ and $V=1.0(\beta=100)$. 
$U=3, V=1$, respectively. In both panels, one also sees that the nonlocal Fock term can be absorbed into the bare dispersion $\varepsilon(\mathbf{k})=-2 t\left[\cos \left(k_{y}\right)+\cos \left(k_{y}\right)\right]$ by defining a $U$ - and $V$-dependent hopping

$$
\tilde{t}(U, V)=t+\delta t(U, V)
$$

We note that standard EDMFT [3-5] does not include the nonlocal Fock term, while $G W+$ EDMFT does. Our finding in this work, and in Refs. [1,2], that the effect of the nonlocal self-energy is to enhance local correlations is valid only if the effect of the nonlocal Fock term on the one-particle dispersion is treated consistently in both formalisms.

We thank Lewin Boehnke for pointing out to us the missing nonlocal Fock term in the $G W+$ EDMFT calculations.

[1] T. Ayral, P. Werner, and S. Biermann, Phys. Rev. Lett. 109, 226401 (2012).

[2] L. Huang, T. Ayral, S. Biermann, and P. Werner, Phys. Rev. B 90, 195114 (2014).

[3] A. M. Sengupta and A. Georges, Phys. Rev. B 52, 10295 (1995).

[4] H. Kajueter, Ph.D. thesis, Rutgers University, 1996.

[5] P. Sun and G. Kotliar, Phys. Rev. B 66, 085120 (2002). 